\title{
MATHEMATICAL MODELING AND ANALYSIS OF MYCOBACTERIUM TUBERCULOSIS TRANSMISSION IN HUMANS WITH HOSPITALIZATION AND REINFECTION
}

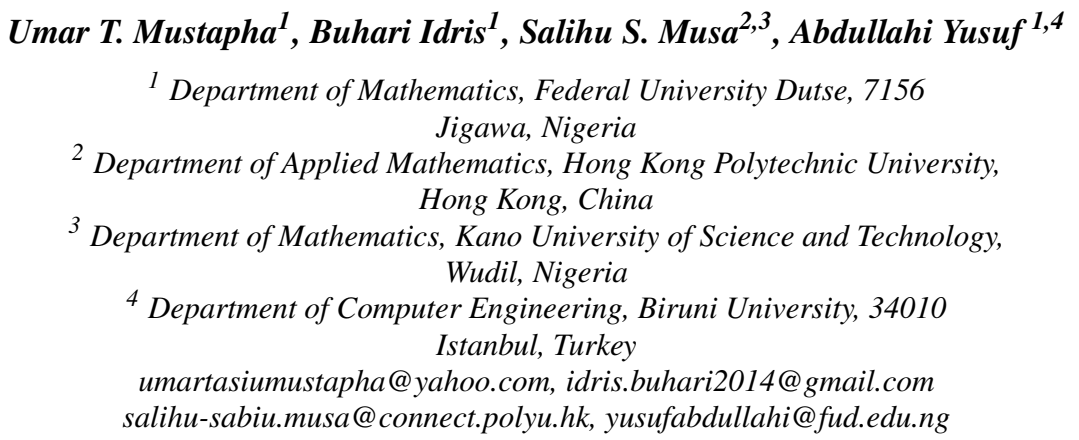

Received: 17 November 2021; Accepted: 18 December 2021

\begin{abstract}
Tuberculosis (TB), a serious public health infection that mainly affects the lungs, is caused by bacteria (Mycobacterium tuberculosis, TB). This research is designed and analyzed using a compartmental modelling approach to study the transmission dynamics of TB with different stages of infection. Qualitative analysis of the proposed model reveals that the model exhibits two equilibrium points: the disease-free equilibrium point (DFE) and the endemic equilibrium (EE). The basic reproduction number $\left(R_{0}\right)$ is determined using the next-generation matrix technique, and stability analysis is carried out to show whether the disease can persist or die out in population. Further analysis of the model shows that the EE is globally asymptotically stable (GAS) when $R_{0}>1$. With the aid of the forward sensitivity index method, we determine the most sensitive parameters of the model to control the spread of TB infection effectively. Our analysis shows that treatment (medication) and campaign awareness coupled with other key control measures, could help maintain the spread of MTB infection in human geographical boundaries.
\end{abstract}

MSC 2010: 92B05, 80A20, 78A60, 35L20

Keywords: Mycobacterium tuberculosis, mathematical modeling, reproduction number, stability analysis, sensitivity analysis

\section{Background of the research work}

Mycobacterium tuberculosis (MTB) is a disease caused by tubercle bacillus or M tuberculosis which belongs to the genus Mycobacterium under the family of Mycobacteriaceae that affect a variety of hosts, including humans, livestock animals (horse, camel, pig) and wild animals (tiger, lion, gorilla), which serve as a reservoir or 
spillover [1]. The disease ranks second to HIV among the leading causes of death worldwide, killing about two million people every year, especially in developing countries, such as India, China, Indonesia, Nigeria, South Africa and so on [1]. In the year 2017 alone, there were about 12.2 million new cases of TB infection globally, including about 1.93 million deaths (and about 0.4 million are people living with HIV) [2]. Previous studies show, that about six countries are considered to have about $60 \%$ of the total MTB cases globally, of which four are counties within Asia, and the rest are African countries [2].

The MTB infection is an air-borne disease which is transmitted from human to human via air [2]. When a human with lung MTB infection coughs, he/she drops the pathogen in the air; an individual is required to inhale a few among the pathogen to get infected with the disease. MTB is classified as pulmonary TB when it affects the lungs, while it is considered extra-pulmonary TB when it affects other parts of the body [1,2]. The most common signs and symptoms of MTB disease are dry coughing, feverish condition, chest pain and most a times loss of weight. MTB appears in the host's body roughly fourteen to eighty-four days (i.e., two to twelve weeks) [1]. The MTB disease can be cured and treated when it has been noticed during the early phase [2]. Active MTB disease can be treated within one hundred and eighty days with non-stop taking of medication (drugs) under the supervision of certified medical personnel trained on handling TB patients. At least 50 million lives were saved during 15 years of TB infection, some of which through treatment, and some via special diagnosis $[1,2]$.

A lot of epidemiological models have been designed and used to assess the transmission dynamics of MTB infection in humans based on geographical disparities (see, for instance, [1,3-6] and the references therein). Some previous studies of epidemic models examined the transmission dynamics of MTB in humans in a confined area/environment, such as transmission of TB infection among prison inmates, and the effect of home and hospital treatment on the overall dynamics of TB infection [7-9]. The aim of the current research work is to design a dynamic model (based on extending some of the research mentioned above) for MTB transmission dynamics to gain more qualitative insight on TB control.

The paper is organised as follows: the model description is contained in section 2 , and section 3 consists of a basic analysis of the model while the sensitivity analysis was carried out in section 4. Finally, the numerical scenario and conclusion are contained in section 5 and 6 respectively.

\section{Description of the model}

The total number of population at time $t$, represented by $N(t)$, is categorised into six (6) compartments as follows: susceptible human, who are at risk of being infected with MTB disease $(S(t))$, Exposed individuals or latent stage $(E(t))$, asymptomatic MTB infected individuals with no clinical symptoms of MTB infection $(A(t))$, symp- 
tomatic MTB infected individuals with clinical symptoms $(I(t))$, hospitalized individuals, who are under treatment of MTB infection $(H(t))$, individuals who recovered from MTB infection $(R(t))$, so that:

$$
N(t)=S(t)+E(t)+A(t)+I(t)+H(t)+R(t) .
$$

The model flow chart (Fig. 1) and the equations for the MTB dynamics are given below.

$$
\begin{aligned}
\frac{d S}{d t} & =\Pi-\lambda S+\theta R-\mu S, \\
\frac{d E}{d t} & =\lambda S-(\sigma+\mu) E, \\
\frac{d A}{d t} & =\alpha \sigma E-\left(\gamma_{1}+\tau_{1}+\delta_{1}+\mu\right) A, \\
\frac{d I}{d t} & =(1-\alpha) \sigma E-\left(\gamma_{2}+\tau_{2}+\delta_{2}+\mu\right) I, \\
\frac{d H}{d t} & =\gamma_{1} A+\gamma_{2} I-\left(\delta_{3}+\tau_{3}+\mu\right) H, \\
\frac{d R}{d t} & =\tau_{1} A+\tau_{2} I+\tau_{3} H-(\theta+\mu) R,
\end{aligned}
$$

where

$$
\lambda=\frac{\beta\left(\eta_{1} E+\eta_{2} H+\eta_{3} A+I\right)}{N},
$$

$\beta$ is the effective contact rate, and $\eta_{1}, \eta_{2}$ and $\eta_{3}$ are modification for for the decrease/increase of MTB infection.

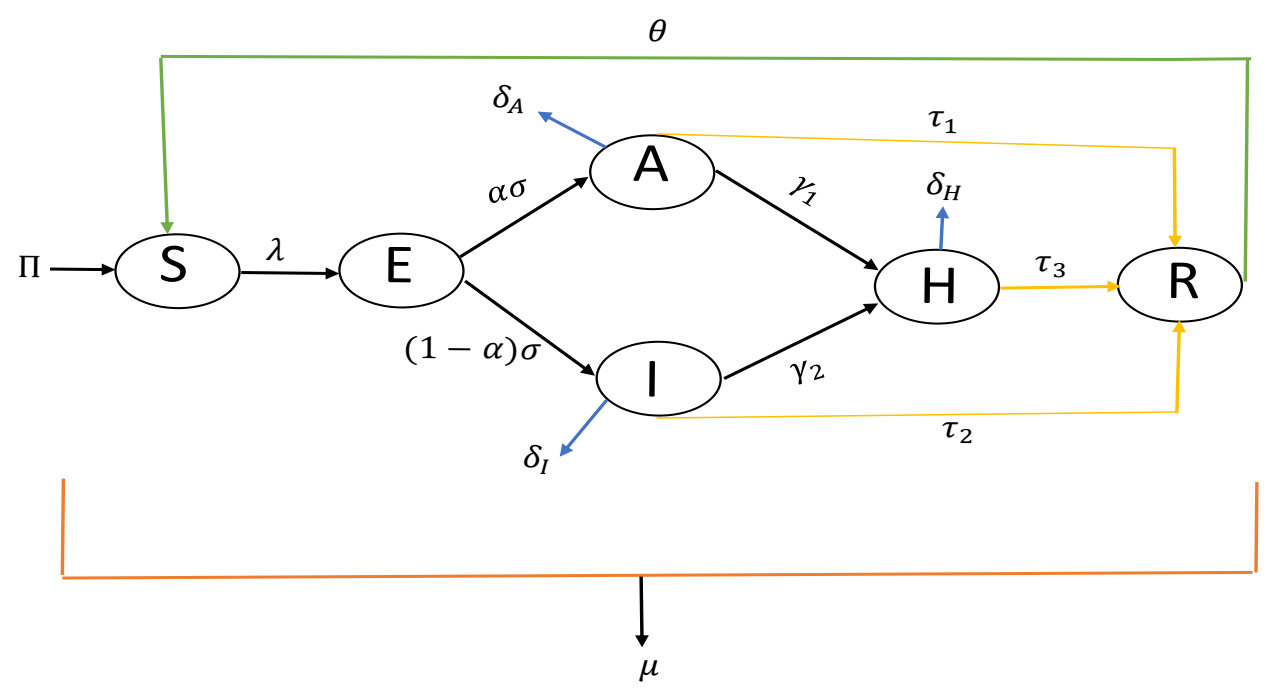

Fig. 1. Flow chart of the MTB infection model 
Table 1. Description of the state variables and parameters apply in the system (1)

\begin{tabular}{r|l}
\hline \multicolumn{1}{r|}{ Variables } & Demonstration \\
\hline$S$ & Total humans population \\
$E$ & Susceptible individuals \\
$A$ & Latent individuals \\
$I$ & Asymptomatically infected individuals \\
$I$ & Syith no clinical symptoms of MTB infection \\
$H$ & with clinical symptoms of MTB infection \\
$R$ & Hospitalized individuals \\
$\Pi$ & Recovered individuals \\
$\mu$ & Recruitment rate of humans \\
$\beta$ & Transmission probability for the MTB infection \\
$\sigma$ & Progression of individuals from latent state of MTB infection \\
$\alpha$ & Proportion of infected individuals who developed symptoms \\
$\theta$ & Loss of immunity \\
\hline Parameters & \\
$\tau_{i}(i=1,2,3)$ & Recovery rates of individuals \\
$\delta_{j}(j=1,2,3)$ & Death caused by the disease \\
$\gamma_{k}(k=1,2)$ & Hospitalization rate of individuals \\
\hline
\end{tabular}

\section{Basic analysis of the model}

\subsection{Positivity of the solution and invariant region}

Theorem 1 The solutions of the system (1) remain positive at initial condition $\forall t>0$. Moreover $\limsup _{t \rightarrow \infty} N(t) \leq \frac{\Pi}{\mu}$.

PROOF Let us consider, the first equation of the system (1) so,

$$
\frac{d S}{d t}=\Pi+\theta R-\lambda S-\mu S \geq-(\lambda+\mu) S
$$

So that

$$
S(t) \geq S(0) \exp ^{-\int_{0}^{t}(\lambda+\mu) d t}>0 .
$$

Similarly, one can see that all the remaining variables of the system are positive $\forall t>0$.

Lemma 1 Let the feasible biological region of the system be (1)

$$
D=\left\{(S, E, A, I, H, R) \in \mathfrak{R}_{+}^{6}: N \leq \frac{\Pi}{\mu}\right\}
$$

are positively, invariant region and also attracting. 
PROOF It is from the fact that $\frac{d N(t)}{d t}=\Pi-\mu N-\left(\delta_{A} A+\delta_{I} I+\delta_{H} H\right) \leq \Pi-\mu N$, so that, $\frac{d N(t)}{d t}<0$, if $N(t)>\frac{\Pi}{\mu}$. Hence, standard comparison theorem [10] are used to prove that $N(t) \leq N(0) \exp ^{-\mu t}+\frac{\Pi}{\mu}\left(1-\exp ^{-\mu t}\right)$. Specifically, $N(t) \leq \frac{\Pi}{\mu}$ if $N(0) \leq \frac{\Pi}{\mu}$. So, $D$ is positive invariant region. Moreover, if $N(t)>\frac{\Pi}{\mu}$ the solution move into $D$ infinitely many time, or $N(t)$ moves closer to $\frac{\Pi}{\mu}$ then variables that are infected move closer to zero. So $D$ is attracting (The solutions are all in $\Re_{+}^{6}$ and finally, $D$ stays or approaches the region).

Consequently, the system (1) is mathematically well-posed and epidemiologically reasonable because the variables are positive for all $t \geq 0$. Thus, it is enough to consider the changes in the system (1) in $D$ [11].

\subsection{Disease-free equilibrium and basic reproduction number}

In the absence of the disease, this means that $\left(E^{*}=0, A^{*}=0, I^{*}=0, H^{*}=0\right)$, therefore the system above becomes

$$
G^{\circ}=\left(S^{*}, E^{*}, A^{*}, I^{*}, H^{*}, R^{*}\right)=\left(\frac{\pi}{\mu}, 0,0,0,0,0\right) .
$$

The stability state of $\varepsilon_{0}$ will be investigated by applying the method of next generation matrix on the system (1) [12]. The matrices $F$ (is the rate of appearance of new infection ) and $V$ (is the matrix containing the rest of the terms)

$$
F=\left[\begin{array}{cccc}
\beta \eta_{1} & \beta \eta_{3} & \beta & \beta \eta_{2} \\
0 & 0 & 0 & 0 \\
0 & 0 & 0 & 0 \\
0 & 0 & 0 & 0
\end{array}\right], V=\left[\begin{array}{cccc}
K_{1} & 0 & 0 & 0 \\
-\alpha \sigma & K_{2} & 0 & 0 \\
-\sigma K_{5} & 0 & K_{3} & 0 \\
0 & -\gamma_{1} & -\gamma_{2} & K_{4}
\end{array}\right]
$$

then $V^{-1}$ is obtained as

$$
V^{-1}=\left[\begin{array}{cccc}
\frac{1}{K_{1}} & 0 & 0 & 0 \\
\frac{\alpha \sigma}{K_{1} K_{2}} & \frac{1}{K_{2}} & 0 & 0 \\
\frac{\sigma K_{5}}{K_{1} K_{3}} & 0 & \frac{1}{K_{3}} & 0 \\
\frac{\sigma\left(\alpha K_{3} \gamma_{1}+K_{2} K_{5} \gamma_{2}\right)}{K_{2} K_{1} K_{3} K_{4}} & \frac{\gamma_{1}}{K_{2} K_{4}} & \frac{\gamma_{2}}{K_{3} K_{4}} & \frac{1}{K_{4}}
\end{array}\right]
$$


and

$$
F V^{-1}=\left[\begin{array}{cccc}
K_{7} & \frac{\beta \eta_{3}}{K_{2}}+\frac{\beta \eta_{2} \gamma_{1}}{K_{2} K_{4}} & \frac{\beta}{K_{3}}+\frac{\beta \eta_{2} \gamma_{2}}{K_{3} K_{4}} & \frac{\beta \eta_{2}}{K_{4}} \\
0 & 0 & 0 & 0 \\
0 & 0 & 0 & 0 \\
0 & 0 & 0 & 0
\end{array}\right]
$$

where,

$$
\begin{aligned}
& K_{1}=\sigma+\mu, K_{2}=\gamma_{1}+\tau 1+\delta_{A}+\mu, K_{3}=\gamma_{2}+\tau_{2}+\delta_{I}+\mu, K_{4}=\delta_{H}+\tau_{3}+\mu, K_{5}= \\
& 1-\alpha \text { and } K_{6}=\theta+\mu, K_{7}=\frac{\beta \eta_{1}}{K_{1}}+\frac{\beta \eta_{3} \alpha \sigma}{K_{1} K_{2}}+\frac{\beta \sigma K_{5}}{K_{1} K_{3}}+\frac{\beta \eta_{2} \sigma\left(\alpha K_{3} \gamma_{1}+K_{2} K_{5} \gamma_{2}\right)}{K_{1} K_{2} K_{3} K_{4}} .
\end{aligned}
$$

It follows that the associated basic reproduction number of the model (1), represented by $R_{0}$, is known as:

$$
R_{0}=\frac{\beta\left(\alpha \sigma K_{3} K_{4} \eta_{3}+\alpha \sigma K_{3} \eta_{2} \gamma_{1}+\sigma K_{2} K_{5} \eta_{2} \gamma_{2}+\sigma K_{2} K_{4} K_{5}+K_{2} K_{3} K_{4} \eta_{1}\right)}{K_{1} K_{2} K_{3} K_{4}}
$$

where $\rho$ represents the spectral radius of $F V^{-1}$. The threshold quantity, $R_{0}$, is the basic reproduction number for MTB infection, which determines whether the disease persists or is eliminated in time [11,13-15].

Using theorem 2 of [12], with reproduction number, $R_{0}<1$, the disease freeequilibrium (DFE) point is locally asymptotically stable, indicating that the population can not be invaded by the disease. Hence, the proof of the following theorem holds as follows.

Theorem 2 The DFE $\left(\varepsilon_{0}\right)$, of the MTB model (1) is locally asymptotically stable (LAS) if $R_{0}<1$, and unstable if $R_{0}>1$.

\subsection{Endemic equilibrium analysis}

Here, the non-continuation of the endemic equilibrium (EE) point of system (1) will be dealt with whenever the $R_{0}$ is less than one. We can say that, the EE point of system (1) is in a stable state, that is, the infection might be wiped out. For $R_{0}>1$, we can say the disease continues to remain in the human population, hence at least one compartment among the compartments of the system (1) is not null. Let $\varepsilon_{1}=\left(S^{*}, E^{*}, A^{*}, I^{*}, H^{*}, R^{*}\right)$ be an endemic equilibrium solution of model (1). Solving the equations of the system (1) with respect to $\lambda$, gives: 


$$
\begin{aligned}
S^{*} & =\frac{K_{1} K_{4} K_{2} K_{3} K_{6} \Pi_{H}}{\left(K_{4} K_{6}(\lambda+\mu) K_{3}-\sigma \theta \lambda K_{5}\left(K_{4} \tau_{2}+\gamma_{2} \tau_{3}\right)\right) K_{2}-\alpha \sigma \theta \lambda K_{3}\left(K_{4} \tau_{1}+\tau_{3} \gamma_{1}\right)}, \\
E^{*} & =\frac{K_{4} K_{2} K_{3} K_{6} \lambda \Pi_{H}}{\left(\left(K_{4} K_{3} K_{6} K_{1}-\sigma \theta K_{5}\left(K_{4} \tau_{2}+\gamma_{2} \tau_{3}\right)\right) K_{2}-\alpha \sigma \theta K_{3}\left(K_{4} \tau_{1}+\tau_{3} \gamma_{1}\right)\right) \lambda+K_{4} K_{2} K_{3} K_{6} K_{1} \mu}, \\
A^{*} & =\frac{\alpha \sigma K_{4} K_{3} K_{6} \lambda \Pi_{H}}{\left(\left(K_{3} K_{6} K_{1}-\sigma \theta K_{5}\left(K_{4} \tau_{2}+\gamma_{2} \tau_{3}\right)\right) K_{2}-\alpha \sigma \theta K_{3}\left(K_{4} \tau_{1}+\tau_{3} \gamma_{1}\right)\right) \lambda+K_{4} K_{2} K_{3} K_{6} K_{1} \mu}, \\
I^{*} & =\frac{K_{5} \sigma K_{4} K_{2} K_{6} \lambda \Pi_{H}}{\left(\left(K_{4} K_{3} K_{6} K_{1}-\sigma \theta K_{5}\left(K_{4} \tau_{2}+\gamma_{2} \tau_{3}\right)\right) K_{2}-\alpha \sigma \theta K_{3}\left(K_{4} \tau_{1}+\tau_{3} \gamma_{1}\right)\right) \lambda+K_{4} K_{2} K_{3} K_{1} \mu}, \\
H^{*} & =\frac{\sigma\left(K_{2} \gamma_{2} K_{5}+K_{3} \gamma_{1} \alpha\right) K_{6} \lambda \Pi_{H}}{\left(\left(K_{4} K_{3} K_{6} K_{1}-\sigma \theta K_{5}\left(K_{4} \tau_{2}+\gamma_{2} \tau_{3}\right)\right) K_{2}-\alpha \sigma \theta K_{3}\left(K_{4} \tau_{1}+\tau_{3} \gamma_{1}\right)\right) \lambda+K_{4} K_{2} K_{3} K_{6} K_{1} \mu}, \\
R^{*} & =\frac{\Pi_{H}\left(K_{5}\left(K_{4} \tau_{2}+\gamma_{2} \tau_{3}\right) K_{2}+\alpha K_{3}\left(K_{4} \tau_{1}+\tau_{3} \gamma_{1}\right)\right) \lambda \sigma}{\left(K_{4} K_{6} K_{1}(\lambda+\mu) K_{3}-\sigma \theta \lambda K_{5}\left(K_{4} \tau_{2}+\gamma_{2} \tau_{3}\right)\right) K_{2}-\alpha \sigma \theta \lambda K_{3}\left(K_{4} \tau_{1}+\tau_{3} \gamma_{1}\right)} .
\end{aligned}
$$

\subsection{Global stability of equilibrium point}

To prove the global stability of the EE points, the following theorem is claimed.

Theorem 3 The EE points, $\varepsilon_{1}$, of the model (1) is globally asymptotically stable $(G A S)$ if $R_{0}>1$ and unstable if $R_{0}<1$.

Proof Suppose the Lyapunov function given by $f=g_{1} E+g_{2} A+g_{3} I+g_{4} H$. Where,

$$
g_{1}=R_{0}, g_{2}=\frac{\beta\left(K_{4}+\eta_{2} \gamma_{2}\right)}{K_{3} K_{4}}, g_{3}=\frac{\beta\left(K_{4}+\eta_{2} \gamma_{1}\right)}{K_{2} K_{4}}, g_{4}=\frac{\beta \eta_{2}}{K_{4}} .
$$

The Lyapunov derivative is known as (dot stand for differentiation with respect to time, t)

$$
\begin{aligned}
& \dot{f}=g_{1} \dot{E}+g_{2} \dot{A}+g_{3} \dot{I}+g_{4} \dot{H}, \\
& =g_{1}\left[\beta\left(\eta_{1} E+A+I+\eta_{2} H\right)-K_{1} E\right]+g_{2}\left(\alpha \sigma E-K_{2} A\right)+g_{3}\left(\sigma K_{5} E-Q_{3} I\right)+g_{4}\left(\gamma_{1} A+\right. \\
& \left.\gamma_{2} I-K_{4} A\right) \\
& =\left(g_{1} \beta \eta_{1}-g_{1} K_{1}+g_{2} \alpha \sigma+g_{3} \sigma G_{5}\right) E+\left(g_{1} \beta-g_{2} K_{2}+g_{4} \gamma_{1}\right) A+\left(g_{1} \beta-g_{3} K_{3}+g_{4} \gamma_{2}\right) I+ \\
& \left(g_{1} \beta \eta_{2}-g_{4} K_{4}\right) H, \\
& \leq\left(R_{0}-1\right)\left(\eta_{1} E+A+I+\eta_{2} H\right) \leq 0 \text { if } R_{0} \leq 1 \text {, since } S \leq N=\frac{\Pi}{\mu} \text { for all } t \in D . \text { Thus, } \\
& \dot{f} \leq 0 \text { if } R_{0} \leq 1 \text { with } \dot{f}=0 \text { if and only if } E=A=I=H=0 . \text { Moreover, the biggest }
\end{aligned}
$$
compact invariant region in $D=\{(S, E, A, I, H, R) \in D: \dot{f}=0\}$ is the singleton $E_{0}$. It shows from the LaSalle's Invariance Principle [16] that each solution of equations (1) with a starting point in $D$ converges to DFE $E_{0}$ as $t \rightarrow \infty$. Substituting $E=A=I=H=0$ into the equation of the model (1), gives $S(t) \rightarrow \frac{\Pi}{\mu}$ as $t \rightarrow \infty$. 
Therefore, $(S, E, A, I, H, R) \rightarrow\left(\frac{\Pi}{\mu}, 0,0,0,0,0\right)$ as $t \rightarrow \infty$ for $R_{0} \leq 1$, so that $E_{0}$ is GAS in $D$ if $R_{0} \leq 1$.

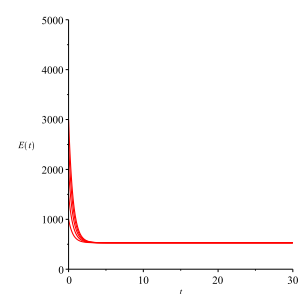

(a)

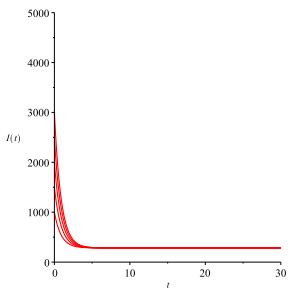

(c)

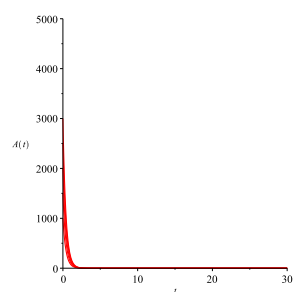

(b)

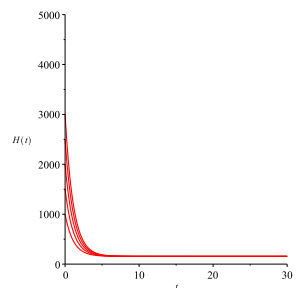

(d)

Fig. 2. Time series plot of the model (1) considering initial condition, using the estimated values (1) with $R_{0}<1$

\section{Sensitivity analysis}

In this section, local sensitivity indexes were evaluated in relation to the reproduction number, $R_{0}$, for the biological parameters of the MTB infection. The model is analyzed by applying the method of forward sensitivity index. The optimization of the projected result is obtained by investigating the impacts and status of key control parameters and their relative effects on the control of the infection [17-19].

It is indicated by $\Gamma_{\beta}^{R_{0}}$ the normalized sensitivity index of the output $R_{0}$ with respect to a parameter $(\theta)$, and is defined as

$$
\dot{\chi_{\theta}}=\Gamma_{\theta}^{R_{0}}=\frac{\theta}{R_{0}} \times \frac{\partial R_{0}}{\partial \theta} .
$$

Using the parameter values defined above $\Pi=53, \mu=0.0047, \beta=0.000535$, $\sigma=0.001, \quad \alpha=0.00071, \quad \tau_{1}=0.000453, \quad \tau_{2}=0.000543, \quad \tau_{3}=0.000234$, $\gamma_{1}=0.2849, \gamma_{2}=0.22806, \delta_{1}=0.0002, \delta_{2}=0.0003, \theta=0.00271, \delta_{3}=0.0004$, the elasticity index values have been calculated (see Table 2 and Fig. 3).

From the elasticity investigation conducted above, one can see that if $\theta$ increases let say by $5 \%$ with the remaining parameters fixed, it attracts another $5 \%$ in $R_{0}$. Similarly, an increase or decrease of any biological parameter of the model (1) will cause the increase or decrease in the reproduction number. 
Table 2. Forward Normalized Sensitivity Indices

\begin{tabular}{|c|c|c|}
\hline Parameter & Elasticity Indices & Elasticity index values \\
\hline$\beta$ & $\dot{\chi}_{\beta}$ & 0.470 \\
$\Pi$ & $\chi_{\Pi}$ & 0.023 \\
$\mu$ & $\dot{\chi}_{\mu}$ & 0.500 \\
$\theta$ & $\dot{\chi}_{\theta}$ & 0.320 \\
$\sigma$ & $\dot{\chi}_{\sigma}$ & 0.011 \\
$\alpha$ & $\dot{\chi}_{\alpha}$ & 0.015 \\
$\gamma_{1}$ & $\dot{\chi}_{\gamma_{1}}$ & 0.543 \\
$\gamma_{2}$ & $\dot{\chi}_{\gamma_{2}}$ & 0.900 \\
$\gamma_{3}$ & $\dot{\chi}_{\gamma_{3}}$ & -0.600 \\
$\tau_{1}$ & $\dot{\chi}_{\tau_{1}}$ & -0.500 \\
$\tau_{2}$ & $\dot{\chi}_{\tau_{2}}$ & -0.340 \\
$\tau_{3}$ & $\dot{\chi}_{\tau_{3}}$ & -0.150 \\
\hline
\end{tabular}

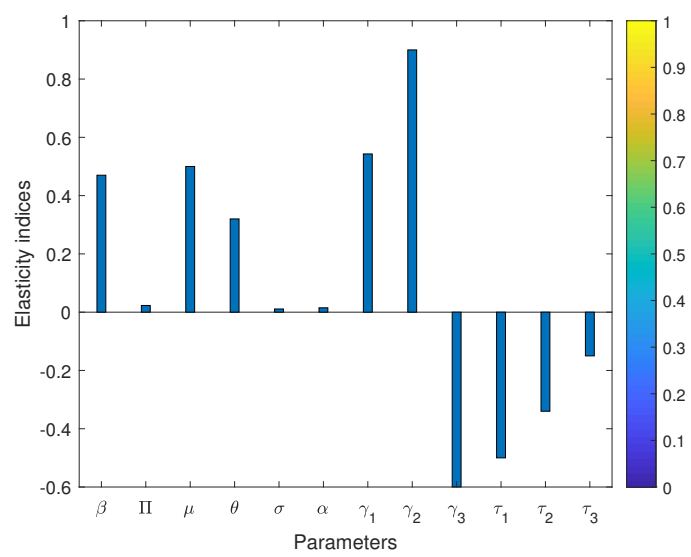

Fig. 3. Elasticity indices with respect to parameters presented in Table 2

\section{Numerical simulations}

Here, we performed numerical simulation for the MTB infection using the model (1). The parameters used are given in Table 1, and the results are demonstrated with control measures. From Figure 4, the susceptible individuals $S(t)$ increase, while exposed individuals decrease $E(t)$, which is plausibly due to the effect of awareness programs. In Figure 5, shows that asymptomatic individuals $A(t)$ and symptomatic individuals $I(t)$ decrease due to medication, campaign awareness and other control measures; a hospitalized compartment, $H(t)$, those who are hospitalized due to infection, decrease due to treatment, and the recovery compartment $R(t)$ increases. 


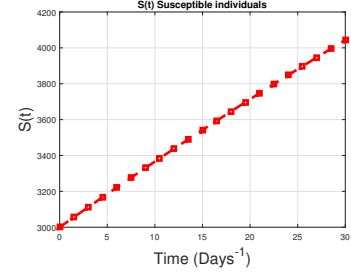

(a)

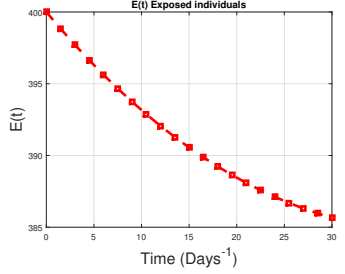

(b)

Fig. 4. Dynamical behaviour of Vulnerable humans $S(t)$ and that of Latent humans $E(t)$ with control

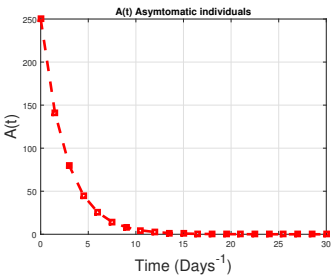

(a)

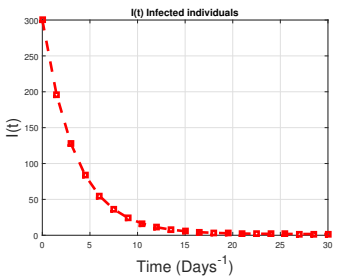

(b)

Fig. 5. Dynamical behaviour of asymtomatic individuals $A(t)$ and that of infected individuals $I(t)$ with control

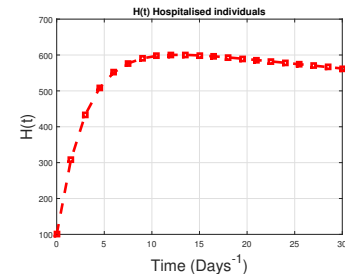

(a)

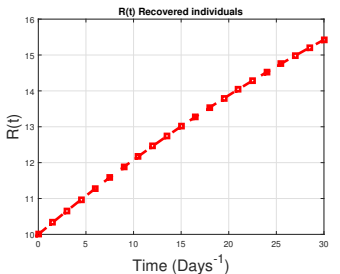

(b)

Fig. 6. Dynamical behaviour of hospitalised individuals $H(t)$ and that of recovered individuals $R(t)$ with control

Table 3. Parameter values for Numerical Simulations

\begin{tabular}{cccc}
\hline Parameter & Values & Units & Source \\
\hline$\Pi$ & 53 & Day $^{-1}$ & Assumed \\
$\mu$ & 0.0047 & Day $^{-1}$ & {$[1]$} \\
$\beta$ & 0.000535 & Day $^{-1}$ & {$[1]$} \\
$\eta_{1}$ & {$[0,1)$} & Dimensionless $^{-1}$ & Assumed \\
$\eta_{2}$ & {$[0,1)$} & Dimensionless $^{-1}$ & Assumed \\
$\theta$ & 0.00271 & Day $^{-1}$ & Est. Using [1] \\
$\tau_{i}(i=1,2,3)$ & $(0.000453,0.000543,0.000234)$ & Day $^{-1}$ & {$[1]$} \\
$\sigma$ & $(0.001)$ & Day $^{-1}$ & {$[20,21]$} \\
$\delta_{j}(j=1,2)$ & $(0.0002,0.0003)$ & Day $^{-1}$ & {$[1]$} \\
\hline
\end{tabular}

\section{Conclusions}

This research work presented an epidemic modelling study for the transmission dynamics of MTB infection in the human population. The model was qualitatively 
analysed to examine the transmission dynamics of MTB disease and was used in curbing the transmission with consideration of different stages of the infection. The system exhibits two equilibrium points: the disease-free equilibrium point (DFE), which is locally asymptotically stable whenever the $R_{0}<1$, otherwise is unstable, and the endemic equilibrium. Numerical results also showed that the treatment, campaign awareness, and other possible measures are vital to control MTB infection in human geographical boundaries.

\section{Acknowledgements}

UTM, BI and AY would like to acknowledge the Federal University Dutse for supporting this work.

\section{References}

[1] Hassan, A.S., Garba, S.M., Gumel, A.B., \& Lubuma, J.S. (2014). Dynamics of Mycobacterium and bovine tuberculosis in a human-buffalo population. Computational and Mathematical Methods in Medicine, 2014, article ID 912306.

[2] World Health Organization (WHO). Tuberculosis, Key Facts. 2021. (https://www.who.int/newsroom/fact-sheets/detail/tuberculosis). Access 6 December 2021.

[3] AlMatar, M., AlMandeal, H., Var, I., Kayar, B., \& Köksal, F. (2017). New drugs for the treatment of Mycobacterium tuberculosis infection. Biomedicine \& Pharmacotherapy, 91, 546-558.

[4] Aparicio, J.P., Castillo-Chavez, C. (2009). Mathematical modelling of turberculosis epidemics. Math. Bios. and Eng., 6, 209-237.

[5] Ozcaglar, C., Shabbeer, A., Vandenberg, S.L., Yener, B., \& Bennett, K.P. (2012). Epidemiological models of Mycobacterium tuberculosis complex infections. Mathematical Biosciences, 236(2), 77-96.

[6] Wahid, B.K.A., \& Bisso, S. (2016). Mathematical analysis and simulation of an age-structured model of two-patch for tuberculosis (TB). Applied Mathematics, 7(15), 1882.

[7] Bowong, S., \& Kurths, J. (2012). Modeling and analysis of the transmission dynamics of tuberculosis without and with seasonality. Nonlinear Dynamics, 67(3), 2027-2051.

[8] Cardona, P.J., Català, M., \& Prats, C. (2020). Origin of tuberculosis in the Paleolithic predicts unprecedented population growth and female resistance. Scientific Reports, 10(1), 42.

[9] Huo, H.F., \& Zou, M.X. (2016). Modelling effects of treatment at home on tuberculosis transmission dynamics. Applied Mathematical Modelling, 40(21-22), 9474-9484.

[10] Sadat, R., Agarwal, P., Saleh, R. et al. (2021). Lie symmetry analysis and invariant solutions of $3 \mathrm{D}$ Euler equations for axisymmetric, incompressible, and inviscid flow in the cylindrical coordinates. Advanced in Difference Equations, 486(2021). DOI: 10.1186/s13662-021-03637-w.

[11] Hethcote, H.W. (2000). The mathematics of infectious diseases. SIAM Review, 42(4), 599-653.

[12] Van den Driessche, P., \& Watmough, J. (2002). Reproduction numbers and sub-threshold endemic equilibria for compartmental models of disease transmission. Mathematical Biosciences, 180(1-2), 29-48.

[13] Anderson, R.M. (1982). Transmission Dynamics and Control of Infectious Disease Agents. In Population Biology of Infectious Diseases (pp. 149-176). Berlin, Heidelberg: Springer.

[14] Anderson, R.M., \& May, R.M. (1992). Infectious Diseases of Humans: Dynamics and Control. Oxford University Press. 
[15] Hussaini, N., Lubuma, J.M., Barley, K., \& Gumel, A.B. (2016). Mathematical analysis of a model for AVL-HIV co-endemicity. Mathematical Biosciences, 271, 80-95.

[16] La Salle, J.P. (1976). The Stability of Dynamical Systems. Society for Industrial and Applied Mathematics.

[17] Mustapha, U.T., Qureshi, S., Yusuf, A., \& Hincal, E. (2020). Fractional modeling for the spread of Hookworm infection under Caputo operator. Chaos, Solitons \& Fractals, 137, 109878.

[18] Usaini, S., Mustapha, U.T., \& Sabiu, S.M. (2018). Modelling scholastic underachievement as a contagious disease. Mathematical Methods in the Applied Sciences, 41(18), 8603-8612.

[19] Wu, J., Dhingra, R., Gambhir, M., \& Remais, J.V. (2013). Sensitivity analysis of infectious disease models: methods, advances and their application. Journal of The Royal Society Interface, 10(86), 20121018.

[20] Wen-Xiu Ma, Mohamed R. Ali, \& Sadat, R. (2020). Analytical solutions for nonlinear dispersive physical model. Complexity, 2020, Article ID 3714832, 8 pages, DOI: 10.1155/2020/3714832.

[21] Cohen, T., Colijn, C., Finklea, B., \& Murray, M. (2007). Exogenous re-infection and the dynamics of tuberculosis epidemics: local effects in a network model of transmission. Journal of the Royal Society Interface, 4(14), 523-531. 